\title{
FEASIBILITY STUDY OF THIN FILM TUNNEL CATHODES
}

\section{for \\ THE NATIONAL AERONAUTICS AND SPACE ADMINISTRATION \\ LANGLEY RESEARCH CENTER}
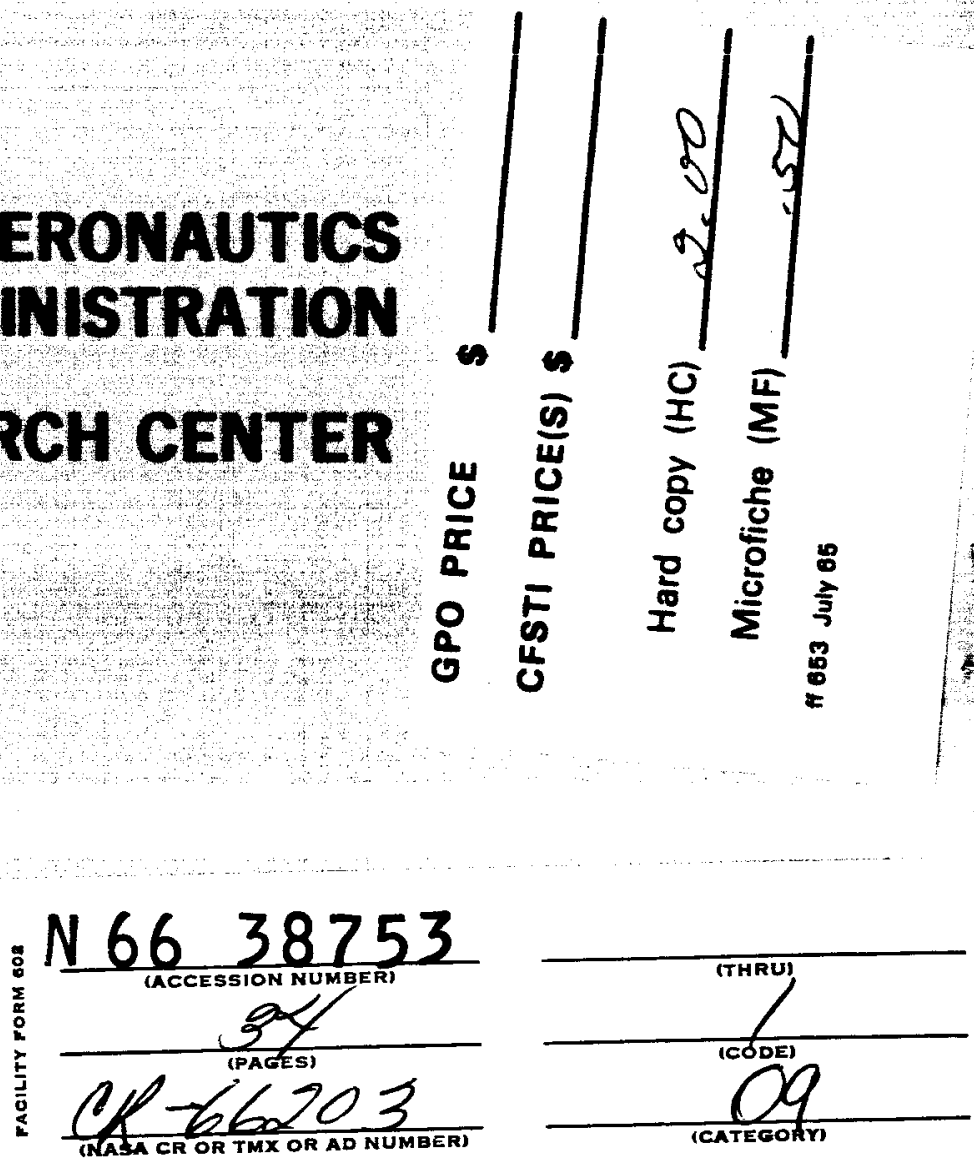

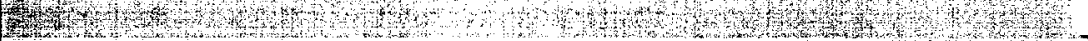

Distribution of this report is provided in the interest of information exchange. Responsibility for the contents resios in the author or organzation that prepered it

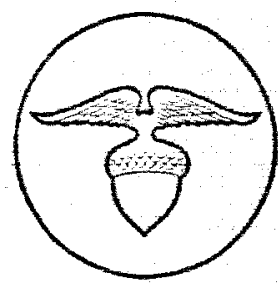

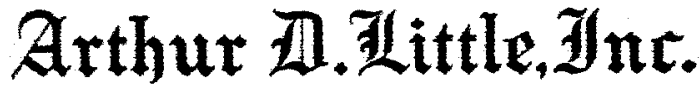


FEASIBILITY STUDY OF THIN FILM TUNNEL CATHODES

by

Donald L. Sullivan and James Shao

Supported by

National Aeronautics and Space Administration

Langley Research Center

Under Contract NAS1-4776

October 1965

C- 67092

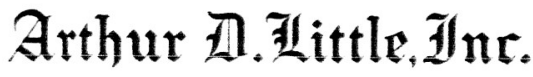
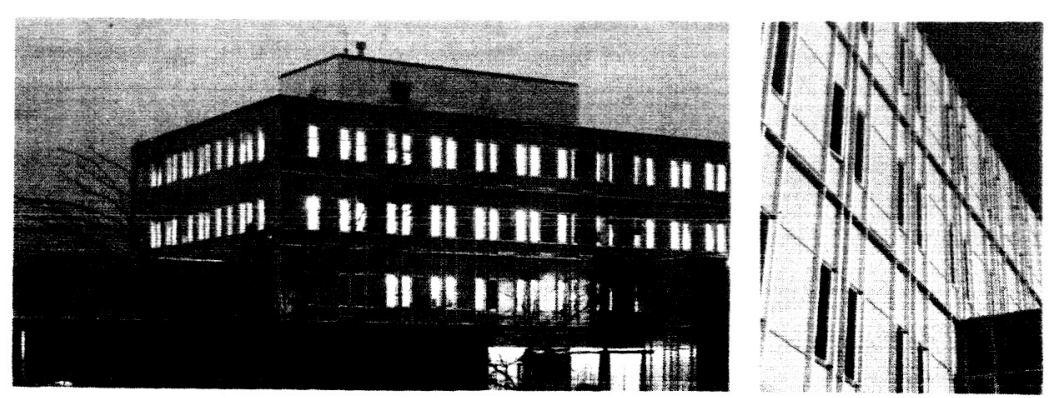
TABLE OF CONTENTS

$\begin{array}{ll}\text { ABSTRACT } & 1\end{array}$

INTRODUCTION 2

EXPERTMENTAL

Cathode Design and Test Apparatus 5

$\mathrm{A} 1 / \mathrm{Al}_{2} \mathrm{O}_{3} / \mathrm{Pt}-\mathrm{Au}$ Cathodes 7

$\mathrm{Al} / \mathrm{Al}_{2} \mathrm{O}_{3} / \mathrm{Pt}$ Cathodes 13

$\begin{array}{ll}\text { THEORETICAL } & 18\end{array}$

$\begin{array}{ll}\text { SUMMARY } & 26\end{array}$

APPENDIX - Plasma Oxidation Techniques 28 


\section{ABSTRACT}

The objective of Phase I of this program has been to investigate the performance of certain types of tunneling cold cathodes to determine their suitability for use in ultra-high vacuum gages. The specific goals were an emission capability of $1 \mathrm{milliamp} / \mathrm{cm}^{2}$, an efficiency of $1 \%$ and a 1 ife expectancy comparable with standard vacuum gage electron sources.

The achievements to date are an emission of $0.25 \mathrm{milliamp} / \mathrm{cm}^{2}$ on a pulse basis with an efficiency of $0.25 \%$ but with a lifetime shortened by a gradual, partially reversible deterioration process which we have not yet been able to identify. 


\section{INTRODUCTION}

The principle of the tunneling cold cathode is illustrated in Figure 1. Electrons from the base metal can penetrate the base metal/ insulator barrier by quantum mechanical tunneling. If the cover metal is very thick, all electrons emitted by the base metal are collected by the cover metal and the structure acts like a "diode" with the nonlinear $I-V$ characteristics shown in Figure $2 a$. If the cover metal is made very thin ( $100 \AA$ or less) some fraction of the electrons injected from the base into the cover metal will retain sufficient kinetic energy to pass through the cover metal and surmount the cover metal/ vacuum barrier. These electrons are the useful emitted current, $I_{e}$ whose dependence on diode voltage $V_{D}$ is shown in Figure $2 b$. The electrons which fail to surmount the cover metal/vacuum barrier constitute the waste current $I_{W}$. The ratio $I_{e} /\left(I_{e}+I_{W}\right)$ or $I_{e} / I_{D}$ is defined as the emission efficiency.

Since the probability of an electron penetrating the base metal/ insulator barrier decreases rapidly with energy below the top of the barrier and the supply of electrons in the base metal decreases rapidly with energy above its Fermi level, almost all of the diode current leaves the base metal at energies within a few tenths of an electron volt of its Fermi level. This narrow (in energy) beam of electrons tunnels into the conduction band of the insulator. If the electrons did not interact with the insulator or the cover metal, they would all be transmitted to the vacuum and the efficiency would be $100 \%$. Unfortunately, strong interactions do occur and present evidence indicates that losses are more severe in the insulator conduction band than in the cover metal. The path shown for the waste electrons in Figure 1 is presumed to be representative of the attenuation suffered by $99.5 \%$ of the injected electrons. This means that the distribution 


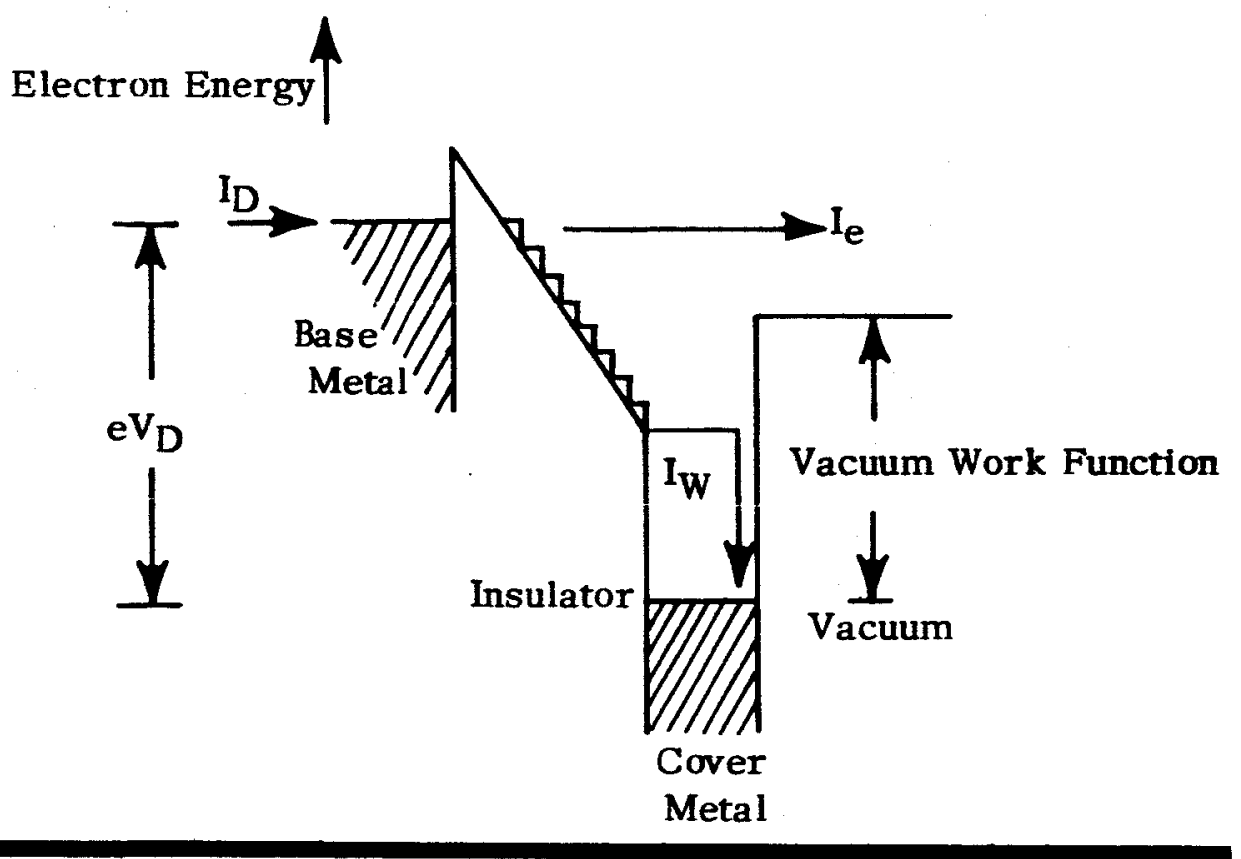

FIGURE 1 TUNNELING COLD CATHODE OPERATING PRINCIPLE

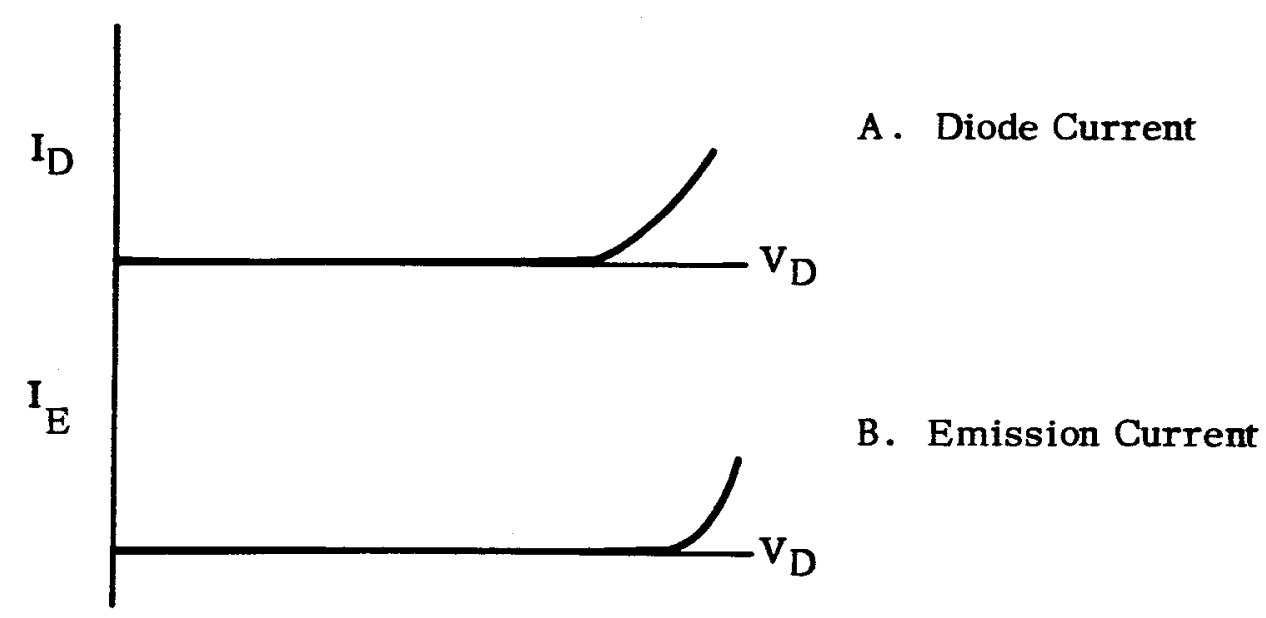

FIGURE 2 TUNNELING COLD CATHODE OPERATING CHARACTERISTICS 
in energy of the electrons at the insulator/cover metal interface will have its maximum at or near the value of the insulator/cover metal barrier height.

Possible means for improving the efficiency are: perfecting the structure of the insulator and cover metal, hopefully improving their electron transmission characteristics; reducing the vacuum work function of the cover metal, so that less energetic electrons can escape; increasing the insulator/cover metal barrier height so as to raise the lower end of the electron energy spectrum and increase the supply of electrons with sufficient energy to surmount the cover metal vacuum barrier. In this phase of the program, we have concentrated on the first and third of these approaches.

\section{EXPERTMENTAL}

\section{Cathode Design and Test Apparatus}

The device configuration chosen for these experiments is shown in Figure 3. Six cathodes are fabricated simultaneously on a $7 / 8^{\prime \prime} \times 1 "$ substrate and can be tested without breaking the vacuum. We have found that carefully handled Corning 7059 glass is far superior to any other insulating substrate and all of our devices were made on this material. The base metal is aluminum, the insulator is aluminum oxide and the cover metal is typically platinum. After the six aluminum bases are deposited they are oxidized by the plasma oxidation technique (see Appendix) to a thickness usually less than $150 \AA$. Next a layer of silicon monoxide or other insulator is deposited over the aluminum bases leaving a $0.1 \mathrm{~cm}^{2}$ window on each, through which the aluminum oxide can be seen. This masks off the aluminum edges which appear to be the source of instabilities observed in earlier structures. A very thin layer of platinum is now applied over the entire area. Its 


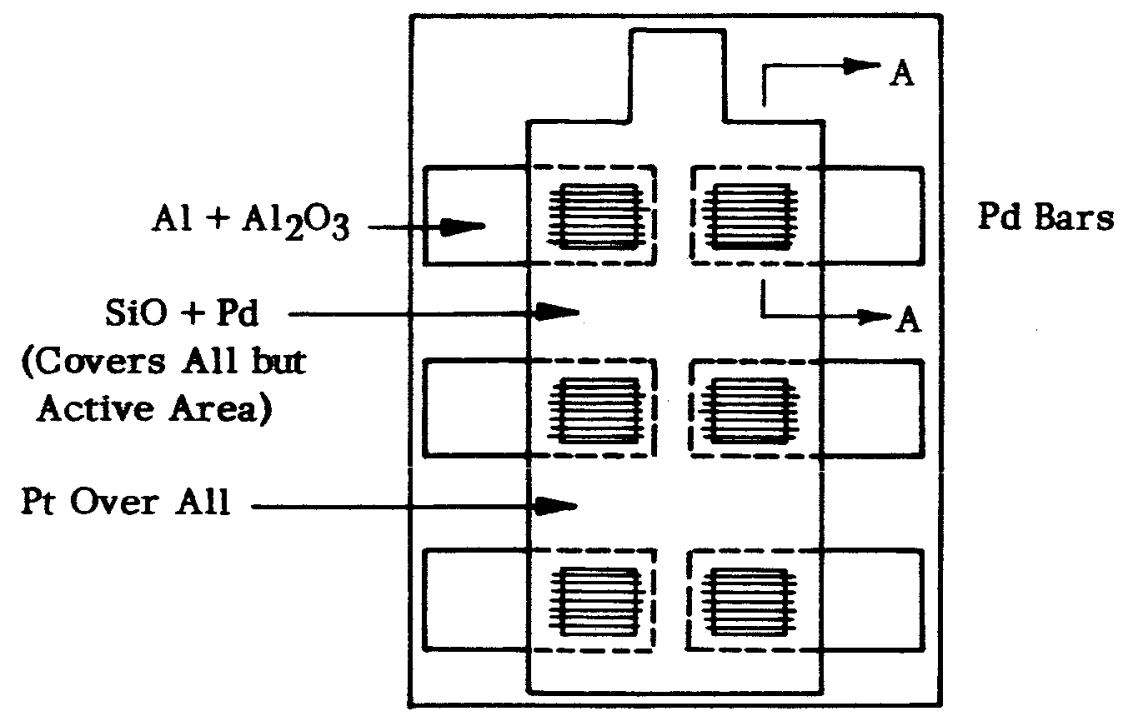

a. Plan View

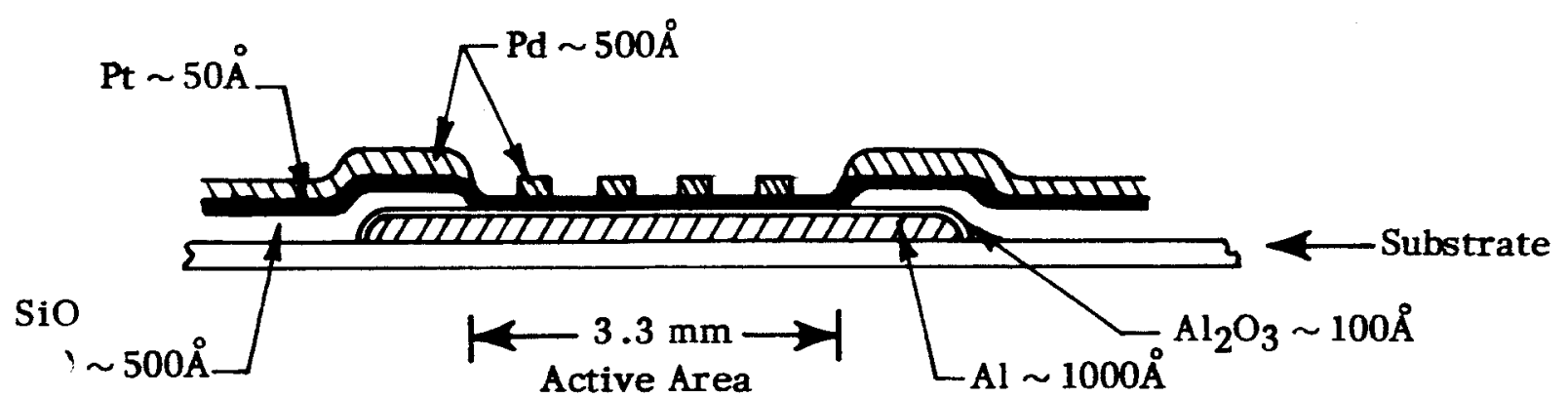

b. Cross Section $\mathbf{A}-\mathbf{A}$

FIGURE 3 EXPERIMENTAL TUNNELING COLD CATHODE 
thickness is very difficult to estimate since resistance measurements are meaningless on such thin films and our quartz crystal thickness monitor reacts strongly to the high source temperatures necessary to evaporate platinum. At present the platinum thickness is controlled by evaporating until a transparent gray film is observed on the glass substrate. Finally a thick coating of palladium or other metal is deposited on top of the platinum to facilitate delivery of current to the active area. This thick coating includes a series of bars that cross the active area and help to make the thin platinum a better equipotential surface in the presence of heavy waste currents.

The completed cathodes are tested in the plexiglass fixture shown in Figure 4. Seven spring loaded pins make contact with the six aluminum bases and the common cover metal. The brass collector electrode is spaced $1 \mathrm{~mm}$ from the cathode surface and with a collector bias of 90 volts the cathode could emit up to $200 \mathrm{milliamps} / \mathrm{cm}^{2}$ before the collected current becomes space charge limited. The measurement electronics is shown in Figure 5 and consists of a transistor ramp generator and a Tektronix $536 \mathrm{X}-\mathrm{Y}$ oscilloscope. The vertical display is chopped between $I_{D}$ and $I_{e}$ at a $100 \mathrm{KC}$ rate.

\section{$\mathrm{A} 1 / \mathrm{Al}_{2} \mathrm{O}_{3} / \mathrm{Pt}-\mathrm{Au}$ Cathodes}

One of the specific aims in this study was to investigate the effect on the emission efficiency of $\mathrm{Al} / \mathrm{A}{ }_{2} \mathrm{O}_{3} / \mathrm{Au}$ cathodes of inserting a very thin layer of platinum between the gold and the oxide. Figure 6 summarizes our findings. Of the six cathodes on the substrate, four were made using thin gold as a cover metal while the other two had the same amount of gold plus a very thin platinum layer. The emission efficiency, $I_{e} / I_{D}$, is indeed higher by a factor of two for the Pt-Au cathode in spite of the greater total cover metal thickness. However, the characteristics are very erratic and this we find to be typical of 

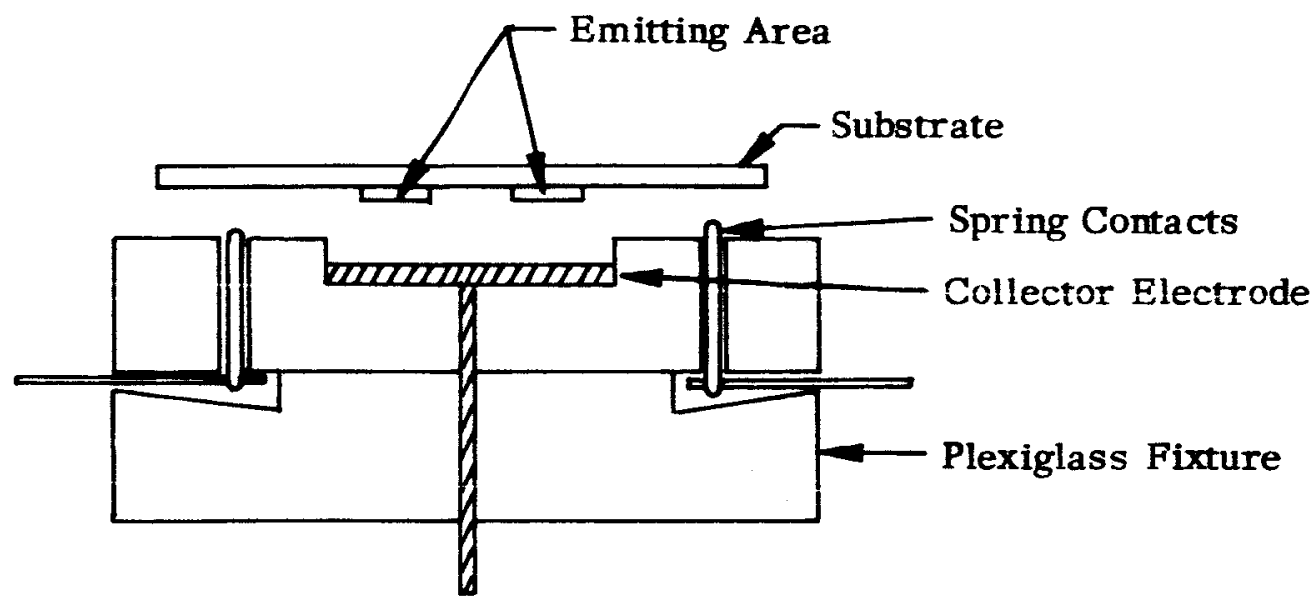

FIGURE 4 CATHODE TEST FIXTURE

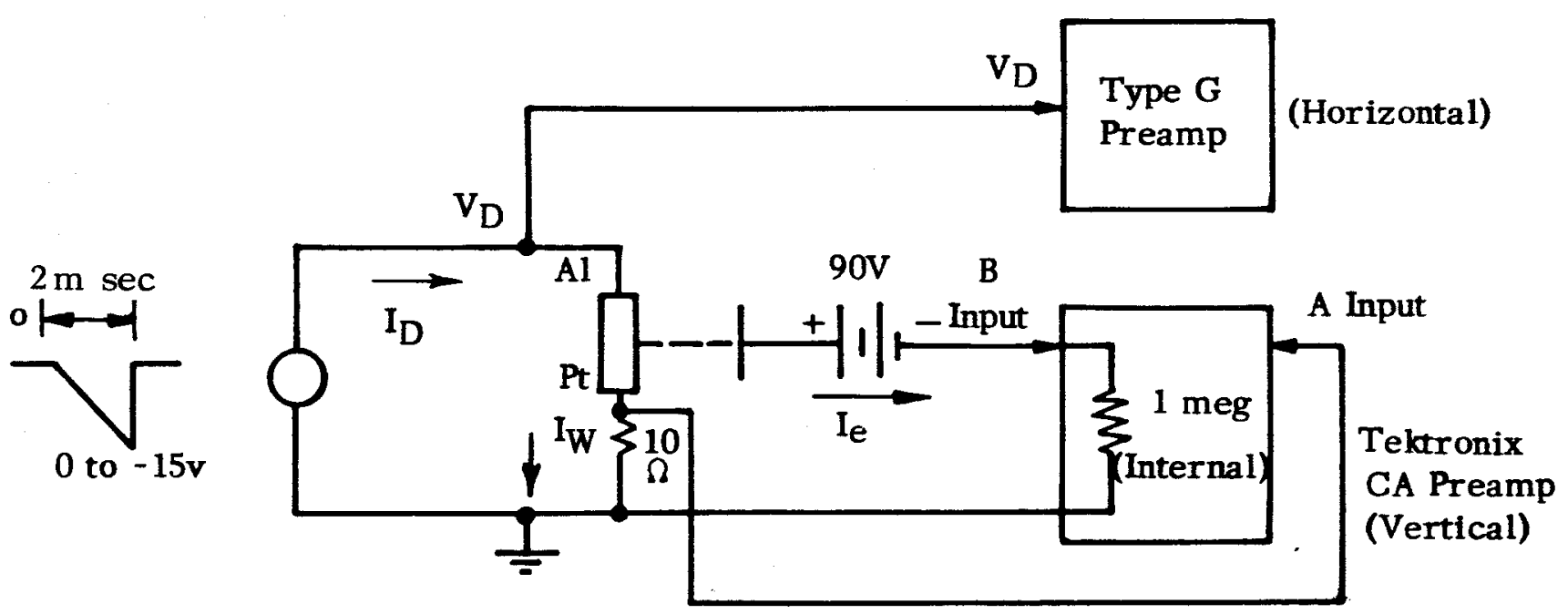

FIGURE 5 CATHODE TEST ELECTRONICS 


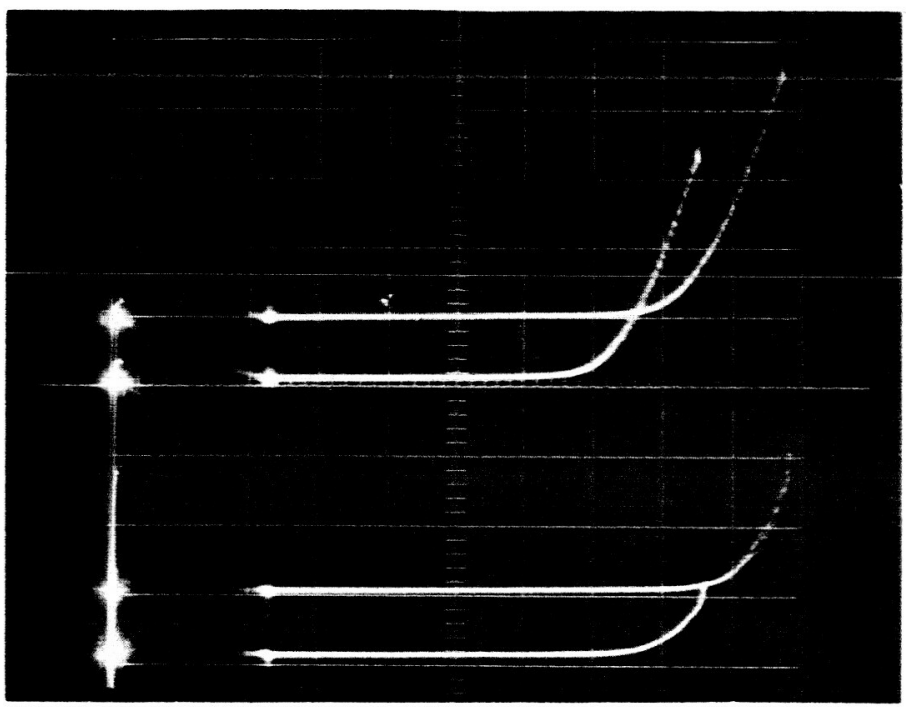

$$
\mathrm{v}_{\mathrm{D}}=0
$$

$$
\mathrm{v}_{\mathrm{D}}=5 \mathrm{v}
$$$$
\mathrm{Al} / \mathrm{Al}_{2} \mathrm{O}_{3} / \mathrm{Au}-
$$

$\mathrm{Al} / \mathrm{Al}_{2} \mathrm{O}_{3} / \mathrm{Pt}-\mathrm{Au}$

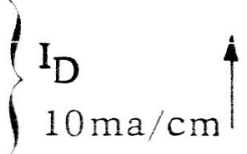

$1 \mathrm{I}_{\mathrm{E}}$ $5 \mu \mathrm{a} / \mathrm{cm}$

\section{FIGURE 6 INCREASE IN EMISSION EFFICIENCY DUE TO THIN}

Pt LAYER BETWEEN $\mathrm{Al}_{2} \mathrm{O}_{3}$ AND Au--Au Grid and $\mathrm{As}_{2} \mathrm{~S}_{3}$ Masking Insulator--Area $10^{-1} \mathrm{~cm}^{2}$--Plasma Oxidized for 20 Minutes with $\mathrm{Al}$ Floating and 10 Minutes with $\mathrm{Al}$ at +3 Volts. 
$\mathrm{Al} / \mathrm{Al}_{2} \mathrm{O}_{3} / \mathrm{Au}$ diodes whose oxide layer is thick enough to exhibit the six volt minimum turnover voltage required for electron emission. Even the thinner $\mathrm{Al} / \mathrm{A} 1_{2} \mathrm{O}_{3} / \mathrm{Au}$ diodes are less robust than their $\mathrm{Al} / \mathrm{A} 1_{2} \mathrm{O}_{3} / \mathrm{A} 1$ counterparts and we conclude that gold is an unsuitable material for these structures.

During our attempts to refine the oxidation process, we performed a considerable number of experiments on $\mathrm{Al} / \mathrm{Al}_{2} \mathrm{O}_{3} / \mathrm{Al}$ diodes. Our findings are summarized in Figure 7 which indicates the range of $I-V$ characteristics achievable in such diodes by the plasma oxidation process. In all three cases the substrate holder was cooled through contact with a liquid nitrogen reservoir. The characteristics of Figure $7 a$ and $b$ were obtained by oxidizing for 5 and 20 minutes respectively with the aluminum floating while that of $7 \mathrm{c}$ was obtained by oxidizing with the aluminum floating for twenty minutes, then at +3 volts for a few minutes and finally at +6 volts for a few more minutes. The very thin oxide of Figure $7 a$ can carry very large currents which in fact seem to be limited by dissipation in the connecting metal strips rather than the oxide itself. Appreciable current flow in the diode of Figure $7 \mathrm{~b}$ starts at a higher voltage but the ultimate current carrying capability is much less than that for the thin oxides. For the relatively thick oxide (probably about $120 \AA$ ) of Figure 7c, the current flow begins at a still higher voltage but the diode can only carry a minute current before it shorts out. Thicker oxides than this have a negligible current carrying capacity. We conclude that with aluminum as a cover metal, diodes cannot be made by this process which will carry appreciable currents at voltages greater than $2-1 / 2$ volts. 

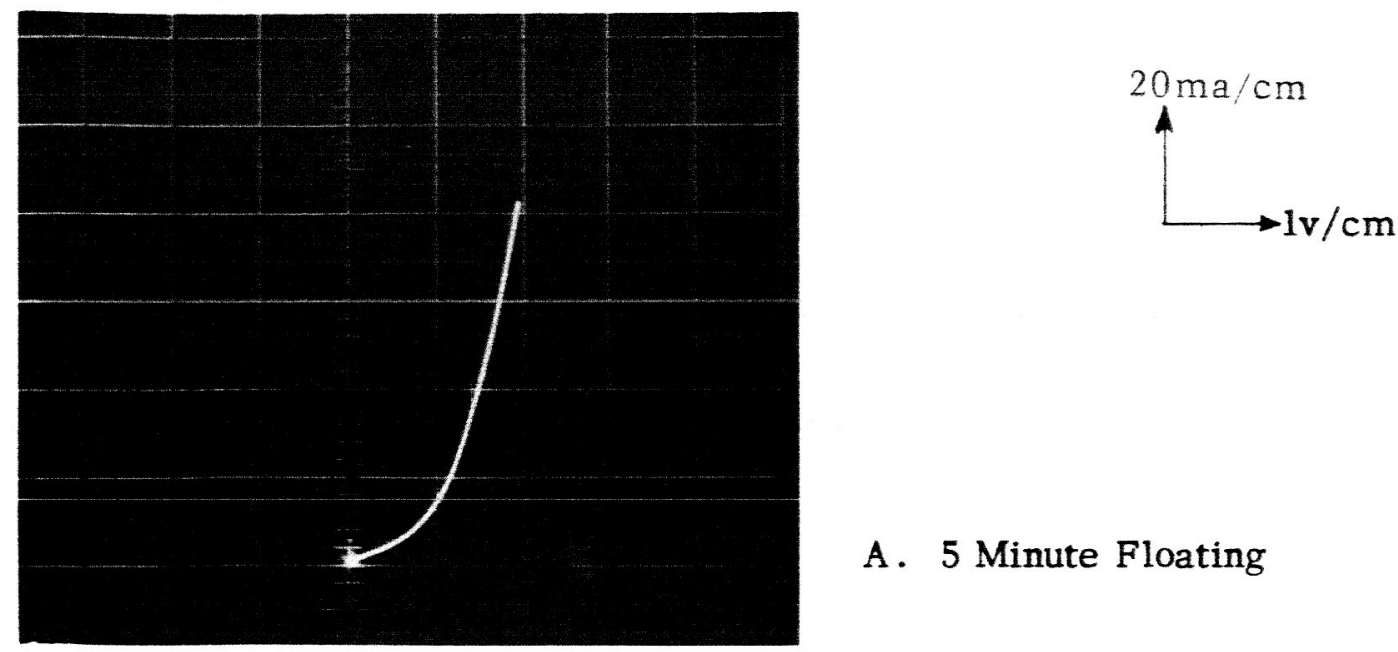

A. 5 Minute Floating
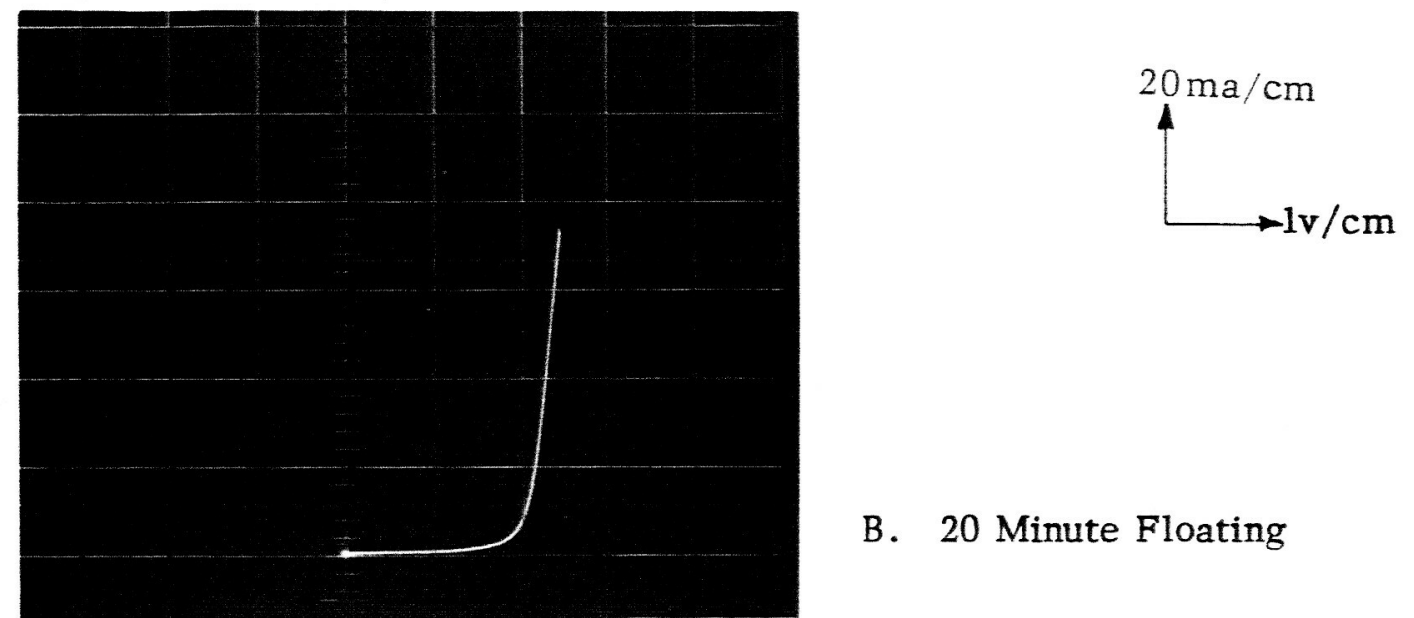

B. 20 Minute Floating
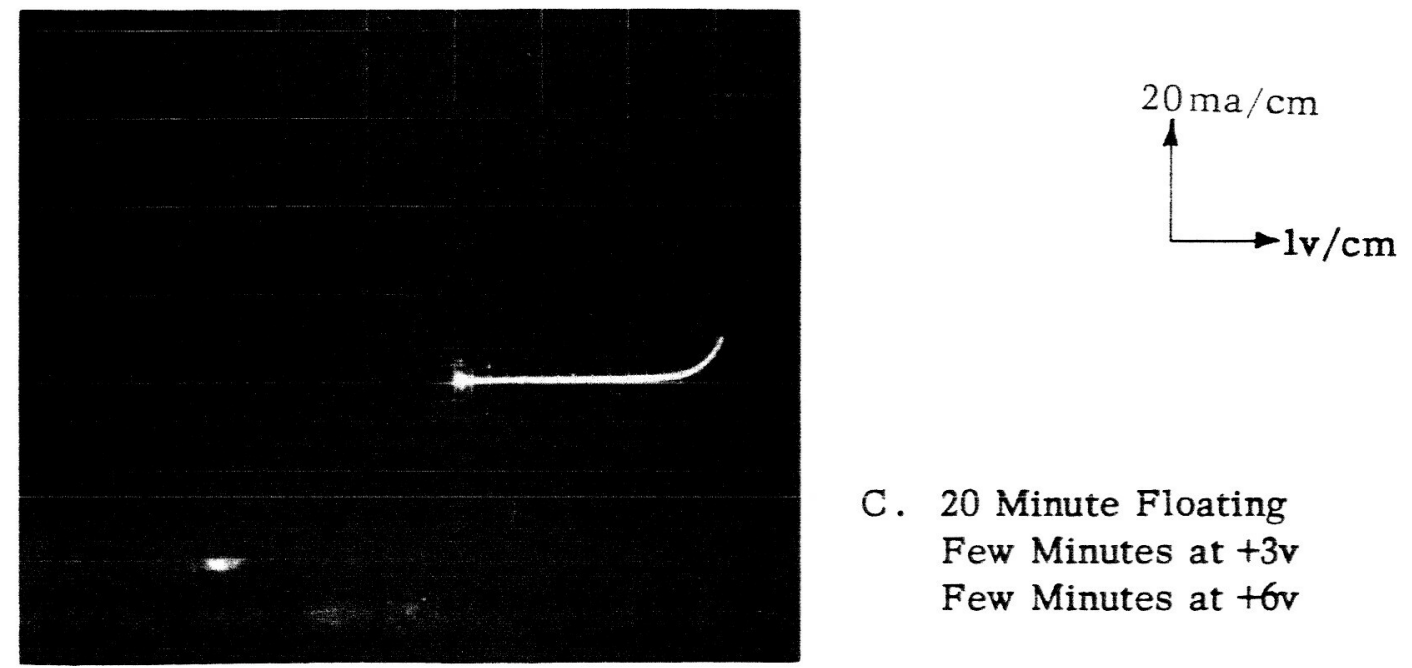

FIGURE 7 RANGE OF I-V CHARACTERISTICS OBTAINABLE $\mathbb{N}$ Al/Al $2 \mathrm{O}_{3} / \mathrm{Al}$ STRUCTURES USING PLASMA OXIDATION TECHNIQUE--Sample Area $10^{-1} \mathrm{~cm}^{2}$ 


\section{$\mathrm{A} 1 / \mathrm{Al}_{2} \mathrm{O}_{3} / \mathrm{Pt}$ Cathodes}

The most promising cathodes we have made so far are those using platinum as a cover metal with palladium bars. While the deterioration to which these cathodes are subject limits their current usefulness, they do have some interesting properties that provide grounds for optimism. They are not only unharmed by exposure to air, but they can be baked to at least $200^{\circ} \mathrm{C}$ in air with dramatic improvement in their characteristics and a partial reversal of the deterioration process. In addition, they are capable of electron emission into air at atmospheric pressure although with an efficiency only about one-tenth that of emission into vacuum.

Figure 8 shows the diode and emission characteristics of such a cathode. A word of explanation is in order about these photographs. Our voltage ramp generator developed a malfunction whereby the ramp became a step about two volts high followed by the continuation of the ramp. Since the first few volts are unimportant, we chose to delay repairs. The resulting photographs show a gap in the I-V characteristics but $V_{D}=0$ is still at the position of the leftmost bright spots.

Figure 8a shows cathode 61-3 just after it was made. The substantial linear rise in diode current at lower voltages is usually characteristic of cathodes which have deteriorated through use but this particular cathode started out that way. The usual new diode characteristic is similar in shape to Figure 6 . Figure $8 \mathrm{~b}$ shows the result of operating the cathode for five minutes at 100 ramps per second. The diode current has acquired a large linear component and the emission current has all but vanished. After baking overnight at $200^{\circ} \mathrm{C}$ in air, the cathode characteristics have returned (Figure 8c) almost to their original shape with the exception of a slight increase in efficiency and a small hump in the diode characteristic which is typical of used cathodes. 


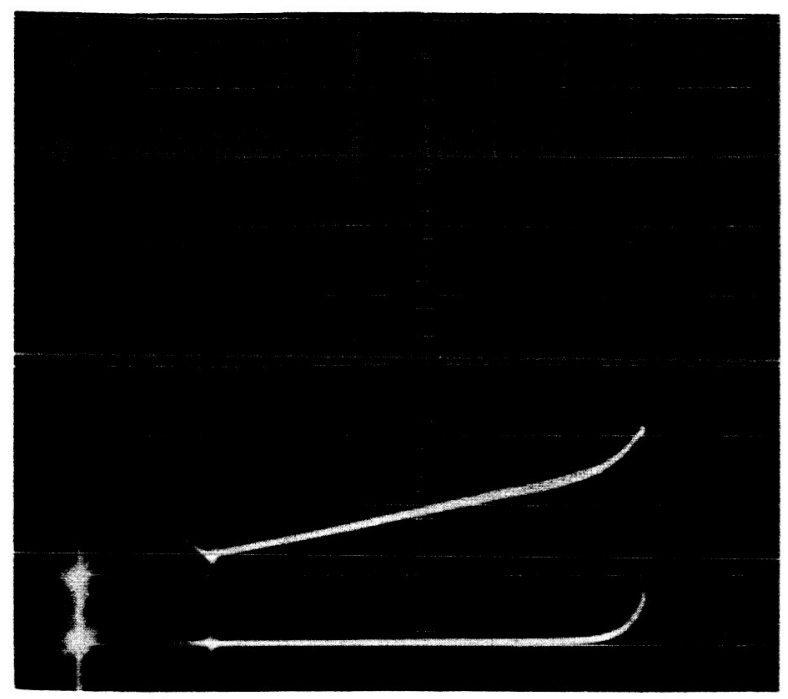

$$
\begin{aligned}
& \mathrm{I}_{\mathrm{D}} \uparrow 10 \mathrm{ma} / \mathrm{cm} \\
& \mathrm{I}_{\mathrm{E}} \uparrow 5 \mathrm{\mu a} / \mathrm{cm} \\
& \mathrm{V}_{\mathrm{D}} \longrightarrow \mathrm{lv} / \mathrm{cm}
\end{aligned}
$$

A. Unused Cathode

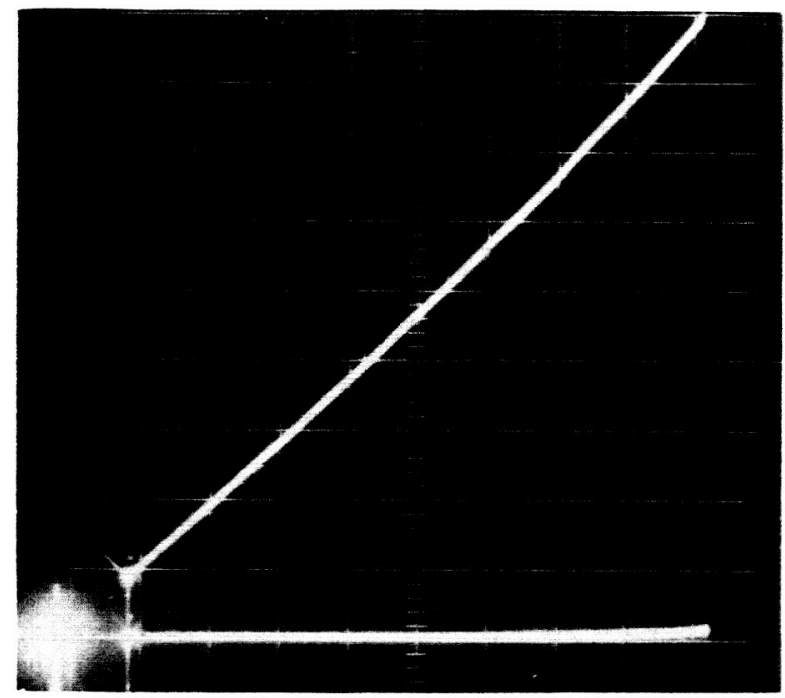

$$
\begin{aligned}
& \mathrm{I}_{\mathrm{D}} \uparrow 10 \mathrm{ma} / \mathrm{cm} \\
& I_{E} \uparrow 5 \mathrm{\mu a} / \mathrm{cm} \\
& \mathrm{v}_{\mathrm{D}} \longrightarrow \mathrm{iv} / \mathrm{cm}
\end{aligned}
$$

B. After 5 Minute Life Test

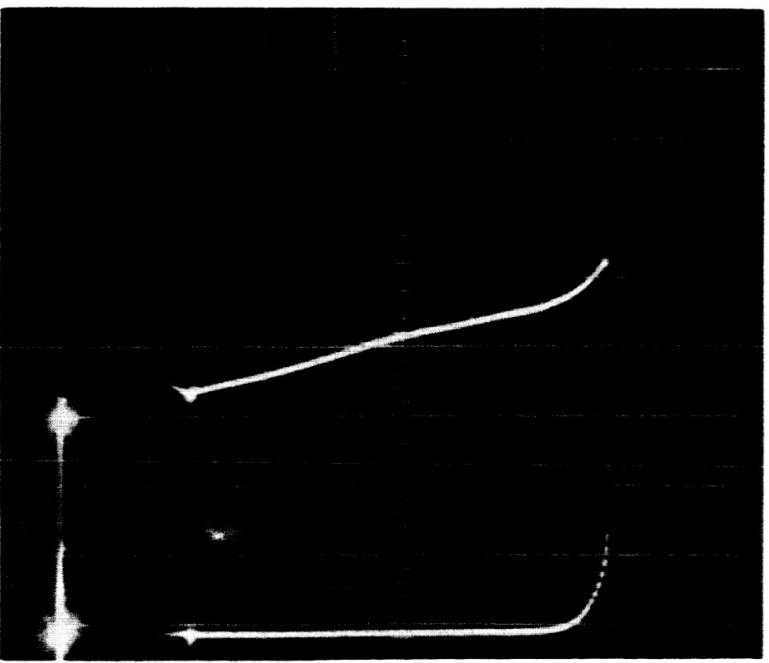

$$
\begin{aligned}
& \mathrm{ID}_{\mathrm{D}} \uparrow 10 \mathrm{ma} / \mathrm{cm} \\
& \mathrm{I}_{\mathrm{E}} \uparrow \quad 5 \mu \mathrm{a} / \mathrm{cm} \\
& \mathrm{V}_{\mathrm{D}} \longrightarrow \mathrm{lv} / \mathrm{cm}
\end{aligned}
$$

FIGURE 8 EFFECT OF LIFE TEST AND BAKING ON CHARACTERISTICS OF $\mathrm{Al} / \mathrm{Al}_{2} \mathrm{O}_{3} / \mathrm{Pt}$ CATHODE 
Figure 9 shows cathode 61-6, on the same substrate as 61-3, before and after the bake. This cathode was not subjected to the five minute 1ife test that wore out 61-3. Its efficiency, defined as $I_{e} / I_{D}$ at constant $V_{D}$, increased from $.025 \%$ to $.25 \%$ at 8 volts. A later cathode subjected to the same treatment not only showed a similar increase in efficiency, but was much more durable as well. While the unbaked cathodes wear out in five minutes, this baked one ran for several hours before deterioration began to occur.

An explanation of this intriguing reversible deterioration would be most useful. The improvement in characteristics after baking is apparently not connected with the rather large field created in the insulator by the contact potential difference between the aluminum and platinum electrodes. One cathode was baked with a 1-1/2 volt bias in the normal operating direction, a second with $1-1 / 2$ volts of the opposite polarity and a third with no applied voltage. In the first cathode, the built in field was reduced from its floating value and probably reversed while in the second cathode the field was considerably increased. If field induced migration of material through the insulator were responsible for the changes, one would expect some difference in the cathode characteristics to result from the above treatment. However, al1 three cathodes behaved identica11y. There is still the possibility that a fairly small field of either polarity is sufficient to sweep mobile impurities out of the insulator and in such a short time that the process could have gone to completion in all three cathodes. A straightforward series of tests can answer this question.

One mechanism that has been proposed for the cathode deterioration is electromigration in the thin platinum film ${ }^{(1)}$. This effect, which has been investigated in a number of metals, involves mass transport in a metal carrying a large unidirectional current by direct momentum exchange between the metal atoms and electrons. It 


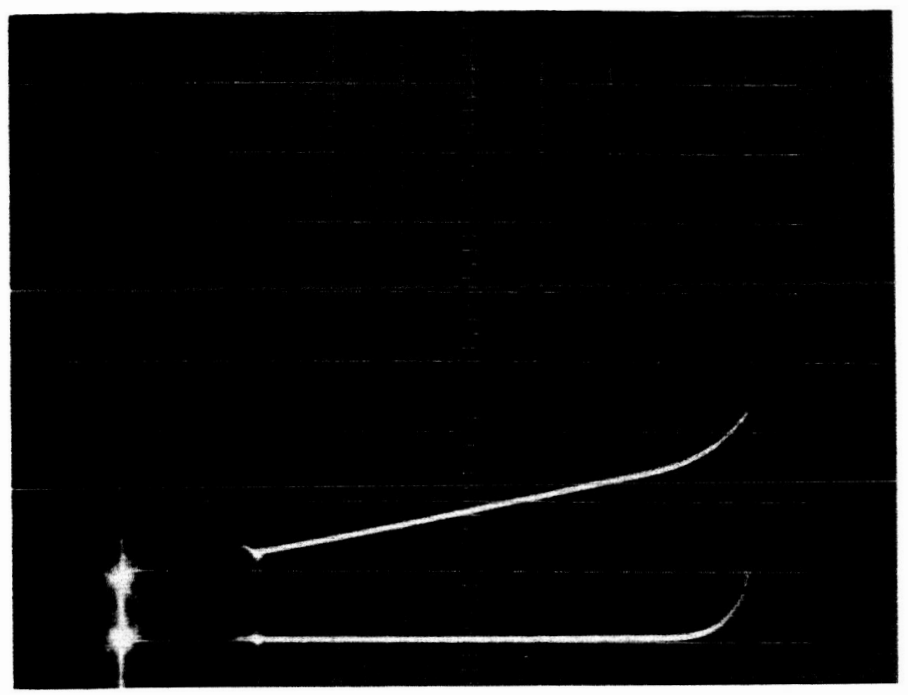

$$
\begin{aligned}
& I_{D} \uparrow 10 \mathrm{ma} / \mathrm{cm} \\
& I_{E} \mid 5 \mu / \mathrm{cm} \\
& \mathrm{v}_{\mathrm{D}} \longrightarrow 1 \mathrm{v} / \mathrm{cm}
\end{aligned}
$$

\section{A. Unused Cathode}

$$
\begin{aligned}
& I_{D} 10 \mathrm{ma} / \mathrm{cm} \\
& I_{E} 5 \mathrm{Ha} / \mathrm{cm} \\
& \mathrm{V}_{D} \longrightarrow 1 \mathrm{v} / \mathrm{cm}
\end{aligned}
$$

B. After Overnight $200^{\circ} \mathrm{C}$ Bake

\section{FIGURE 9 EFFECT OF BAKING ON CHARACTERISTICS OF $\mathrm{Al} / \mathrm{Al}_{2} \mathrm{O}_{3} / \mathrm{Pt}$ CATHODE}


is usually observed at high temperatures but because the platinum film is so thin, surface mobility of significant proportions may occur at lower temperatures. If such an effect is present, it could be neutralized by operating the cathode with current pulses of alternate polarity.

In summary, the $\mathrm{Al}_{1} / \mathrm{Al}_{2} \mathrm{O}_{3} / \mathrm{Pt}$ cathode structure appears to have considerable promise. Baking at $200^{\circ} \mathrm{C}$ in air greatiy improves the electrical wear resistance and can partly rejuvenate worn out cathodes. The deterioration phenomenon is repeatable and well behaved so that a firm foundation exists for the experimental work required to determine its cause. 


\section{THEORETICAL}

Application to evaporated thin film structures of analytical techniques which were evolved to describe single crystal bulk phenomena is a hazardous business at best. The thin films are almost always polycrystalline or amorphous, the structures are sometimes only a few atomic layers thick and are certainly far from having neat plane-parallel geometry. Therefore it is quite gratifying to find that one can of ten use some outrageously simple model for a thin film structure and get good enough agreement between theory and experiment that sensible predictions can be made about the effects on device performance of controllable parameters. The following analysis is made with due regard for its limitations and with the object of determining the sensitivity of the current emitted by tunneling cold cathodes to the barrier heights and charge scattering mechanisms in various parts of the structure.

Figure 10 shows the $x, E_{x}$ projection of the assumed cathode band structure. The various layers are assumed to be parallel infinite planes, the $y$ and $z$ components of things being integrated out where necessary and ignored where possible. The zero of energy is taken at the Fermi level of the aluminum base metal. The result desired from the analysis is $N_{t}(E)$, the distribution in energy of the number of electrons $/ \mathrm{cm}^{2}-\mathrm{sec}$ normally incident on the platinum/vacuum barrier and its integral, the total current which surmounts that barrier.

The first step is the calculation of $N(E)$, the current density distribution along the bottom of the insulator conduction band. Two major assumptions are made; first, the scattering mechanism for electrons in the insulator is such that an electron loses all of its $x$ directed kinetic energy in a collision. Second, the scattering is proportional to the number of electrons present in a given space and energy interval. The latter leads to an exponential survival law whose characteristic parameter is an attenuation length, L(E'), which is generally energy dependent but which we will assume to be constant. 


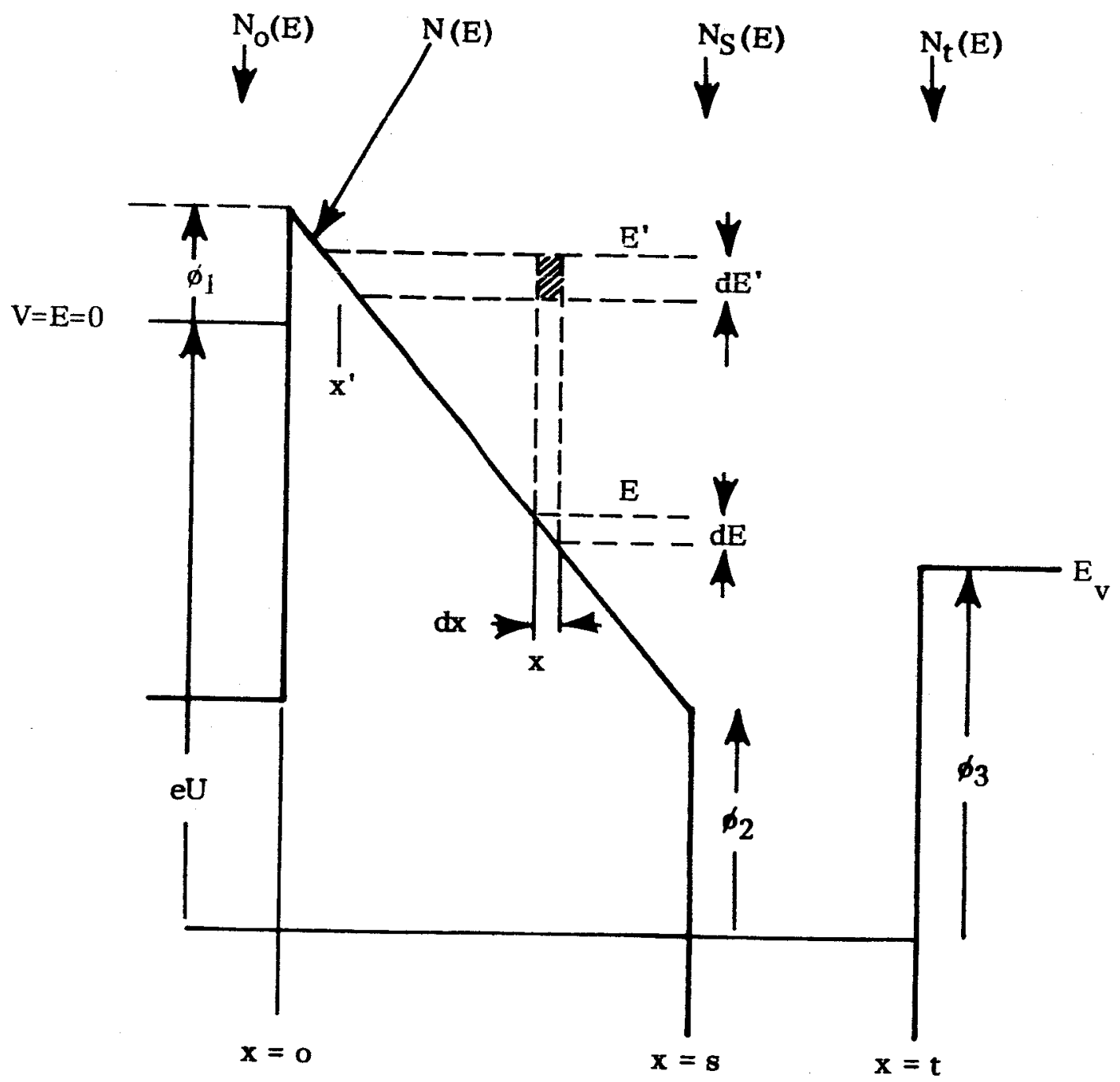

Al

$\mathrm{Al}_{2} \mathrm{O}_{3}$

Pt

Vacuum

FIGURE 10 ENERGY BAND STRUCTURE ASSTJMED FOR TUNNELING COLD CATHODE 
The number of electrons/ $\mathrm{cm}^{2}-\mathrm{sec}$ approaching the metal/oxide barrier at $\mathbf{x}=0$ with $x$ component of kinetic energy between $E_{x}$ and $E_{x}+$ $\mathrm{dE}_{\mathrm{x}}$ is equal to the density of such electrons ${ }^{(2)}$ times their velocity. Writing $E$ for $E_{x}$ henceforth

$N_{0}(E)=\frac{4 \pi m k T}{h^{3}} \ln \left\{\exp \left(\frac{-E}{k T}\right)+1\right\} \frac{\text { electrons }}{c^{2}-\text { sec-erg }}$

This current impinges on the barrier and has a probability of transmission, using the WKB approximation of ${ }^{(3)}$

$T(E)=\exp \left\{-\frac{8 \pi \sqrt{2 m}}{3 h}\left(\frac{s}{e U+\emptyset_{1}-\emptyset_{2}}\right)\left(\emptyset_{1}-E\right)^{3 / 2}\right\}$

The product $N_{0}(E) T(E)=P_{0}(E)$ represents the component of the current at the conduction band bottom due to electrons tunneling directly from the aluminum. A more important contribution is due to electrons which have tunneled into the oxide at some higher energy E' and have been accelerated by the field until they suffer a collision and fall to the conduction band bottom at energy $\mathbf{E}$. The total current density at energy $E$ is thus

$$
N(E)=P_{0}(E)+\int_{E}^{\infty}\left[\text { Fa11out from } E^{\prime}\right] d^{\prime}
$$

The fallout can be computed by assuming that $N\left(E^{\prime}\right)$ is known. Then the number of electrons per $\mathrm{cm}^{2}-\mathrm{sec}$ in energy interval $\mathrm{dE}^{\prime}$ which survive without scattering until they reach the position $x$ is

$$
N\left(E^{\prime}\right) e^{-\frac{\left(x-x^{\prime}\right)}{L\left(E^{\prime}\right)}} d E^{\prime}
$$


As a result of the assumed exponential survival law, the number of electrons per $\mathrm{cm}^{2}-\mathrm{sec}$ which are scattered into energy interval $\mathrm{dE}$ is expression (4) times $\frac{d x}{L}$, where $d x$ is the spatial interval associated with the intersection of $\mathrm{dE}$ and the conduction band bottom. Also one can take account of the known slope of the conduction band bottom

$$
\begin{aligned}
V=E & =\emptyset_{1}-\left(\mathrm{eU}+\emptyset_{1}-\emptyset_{2}\right) \frac{\mathrm{x}}{\mathrm{s}} \\
& =\emptyset_{1}-\mathrm{A}(\mathrm{U}) \mathrm{x}
\end{aligned}
$$

to rewrite (4) in terms of energy variables only. Performing these substitutions in the fallout term of (3) leads to the integral equation

$$
N(E)=P_{0}(E)+\int_{E}^{\infty} \frac{e^{-\frac{E^{\prime}-E}{A(U) L\left(E^{\prime}\right)}} N\left(E^{\prime}\right) d E^{\prime}}{A(U) L\left(E^{\prime}\right)}
$$

Now, letting $L(E)$ be constant and making the substitutions

$$
a=\frac{1}{A(U) L}, t=E^{\prime}-E
$$

the equation reduces to

$$
N(E)=P_{0}(E)+a \int_{0}^{\infty} e^{-a t} N(t+E) d t
$$

This can be solved by differentiating with respect to $\mathbf{E}$

$$
\begin{aligned}
\frac{d N(E)}{d E} & =\frac{d P_{0}(E)}{d E}+a \int_{0}^{\infty} e^{-a t} \frac{d N(t+E)}{d E} d t \\
& =\frac{d P_{0}(E)}{d E}+a \int_{0}^{\infty} e^{-a t} \frac{d N(t+E)}{d t} d t
\end{aligned}
$$


because of the formal symmetry of $N(t+E)$ with respect to $t$ and $E$. The integral can now be integrated by parts and equation (7) becomes

$$
\frac{\mathrm{dN}(E)}{\mathrm{dE}}=\frac{\mathrm{dP}_{\mathrm{o}}(E)}{\mathrm{dE}}-a \mathrm{P}_{\mathrm{o}}(E)
$$

This can be directly integrated to

$$
N(E)=P_{0}(E)-a \int P_{0}(E) d E+\text { constant }
$$

$P_{0}(E)=N_{0}(E) T(E)$ is a function which is significant only in the immediate vicinity of the aluminum Fermi level since $N_{0}(E)$ decays rapidly above $E_{F}$ and $T(E)$ decays rapidly below. This makes it plausible to assume that $P_{0}(E)$ is a Dirac delta function although other approximations can be easily used in ( 8 ). So let

$$
P_{0}(E)=B \delta\left(E_{m}-E\right)
$$

where

$$
B=\int_{0}^{\infty} P_{0}(E) d E,
$$

the total number of electrons $/ \mathrm{cm}^{2}-\mathrm{sec}$ injected from the aluminum, and $E_{m}$ is the energy at which the maximum value of the actual $P_{0}(E)$ occurs. Under these conditions the function $N(E)$ becomes

$$
\begin{array}{rlrl}
N(E) & =B \delta\left(E_{m}-E\right)+a B & ; E \leq E_{m} \\
& =0 & & ; E>E_{m}
\end{array}
$$

and looks like

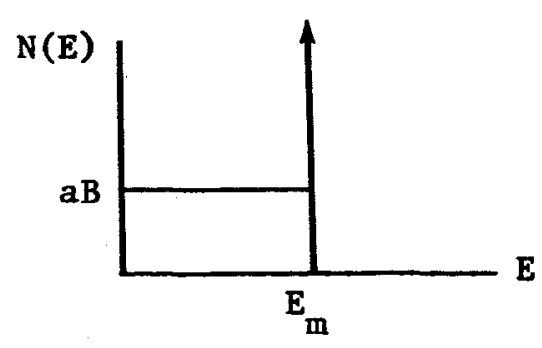


With the fallout process already accounted for one can now write the distribution $\mathrm{N}_{x}(E)$ in energy of the electrons crossing the plane at $x$ as

$$
N_{X}(E)=N(E) e^{-\frac{\left(x-x_{E}\right)}{L}}
$$

where $x_{E}=\frac{\emptyset_{1}-E}{A(U)}$ is the value of $x$ at the intersection of the conduction band bottom with energy $E$. At the entrance to the cover metal, the distribution is

$$
N_{S}(E)=B e^{a\left(\phi_{1}-E\right)} e^{-\frac{S}{L}}\left[\delta\left(E_{m}-E\right)+a\right]
$$

If the attenuation length in the cover metal is $l$ and the scattering in the metal is assumed to remove the electrons from the useful part of the distribution ${ }^{(4)}$ the energy spectrum at the vacuum barrier is

$$
\begin{array}{rlrl}
N_{t}(E) & =B e^{a\left(\phi_{1}-E\right)} e^{-\frac{S}{L}} e^{-\frac{(t-S)}{\ell}}\left[\delta\left(E_{m}-E\right)+a\right] ; E \leq E_{m} \\
& =0 & ; E>E_{m}
\end{array}
$$

Finally, the emitted current is

$$
\begin{aligned}
J_{E} & =e \int_{E_{v}}^{\infty} N_{t}(E) d E \\
& =e B e^{-\frac{S}{L}} e^{-\frac{(t-s)}{l}} e^{\frac{s}{L}\left(\frac{e u+\phi_{1}-\emptyset_{3}}{e u+\emptyset_{1}-\emptyset_{2}}\right)}
\end{aligned}
$$

The injected current $J_{D}=e B$ amps $/ \mathrm{cm}^{2}$ so that the current transfer ratio is 


$$
\alpha=\frac{J_{E}}{J_{D}}=e^{-\frac{(t-S)}{l}} e^{-\frac{s}{L}\left(\frac{\emptyset_{3}-\emptyset_{2}}{\text { eu+ } \emptyset_{1}-\emptyset_{2}}\right)}
$$

Let us estimate $\alpha$ for the following set of parameters, for which Figure 10 is drawn to scale:

$$
\begin{aligned}
& \mathrm{eU}=8 \mathrm{e} \cdot \mathrm{v} . \\
& \emptyset_{1}=1.5 \\
& \emptyset_{2}=3 \\
& \emptyset_{3}=5 \\
& \mathrm{~s} / \mathrm{L}=100 \AA / 5 \AA=20 \\
& \frac{\mathrm{t}-\mathrm{S}}{l}=50 \AA / 50 \AA=1
\end{aligned}
$$

This gives an $\alpha$ of $7.8 \times 10^{-4}$ which is not too far from that obtained from real cathodes. More important for our purposes is an estimate of the relative sensitivity of the current transfer ratio to changes in the parameters. These are calculated below for the parameters 1 isted above.

$$
\frac{\mathrm{d} \ln \alpha}{\mathrm{d}(\mathrm{eU})}=\frac{\mathrm{S}}{\mathrm{L}} \frac{\left(\emptyset_{3}-\emptyset_{2}\right)}{\left(\mathrm{eU}+\emptyset_{1}-\emptyset_{2}\right)^{2}}=\frac{\emptyset_{3}-\emptyset_{2}}{\mathrm{LS}}\left(\frac{1}{\mathrm{~F}^{2}}\right)=+0.9
$$

where $F$ is the field in the insulator.

$$
\frac{\mathrm{d} \ln \alpha}{\mathrm{d} \emptyset_{2}}=\frac{\mathrm{s}}{\mathrm{L}} \frac{\mathrm{eU}+\emptyset_{1}-\emptyset_{3}}{\left(\mathrm{eU}+\emptyset_{1}-\emptyset_{2}\right)^{2}}=\frac{\mathrm{eU}+\emptyset_{1}-\emptyset_{3}}{\mathrm{LS}}\left(\frac{1}{\mathrm{~F}^{2}}\right)=+2.1
$$




$$
\begin{aligned}
& \frac{\mathrm{d} \ln \alpha}{\mathrm{d} \emptyset_{3}}=-\frac{\mathrm{s}}{\mathrm{L}} \frac{1}{\left(\mathrm{eU}+\emptyset_{1}-\emptyset_{2}\right)}=-\frac{1}{\mathrm{LF}}=-3.1 \\
& \frac{\mathrm{d} \ln \alpha}{\mathrm{d}\left(\frac{\mathrm{s}}{\mathrm{L}}\right)}=-\frac{\left(\emptyset_{3}-\emptyset_{2}\right)}{\left(\mathrm{eU}+\emptyset_{1}-\emptyset_{2}\right)}=-0.3 \\
& \frac{\mathrm{d} \ln \alpha}{\mathrm{d}\left(\frac{\mathrm{t}-\mathrm{S}}{\ell}\right)}=-1
\end{aligned}
$$

This says that the most sensitive parameter is the cover metal/vacuum barrier but that its influence is less at higher insulator fields. The sensitivity to $\emptyset_{2}$, which was the original object of this investigation, turns out to be also quite large. An increase of $0.5 \mathrm{e} . \mathrm{v}$. in $\emptyset_{2}$ will double the value of $\alpha$. Furthermore, an increase in $\phi_{2}$ will allow a larger value of eU for the same insulator field, giving a double benefit.

These expressions are useful for getting a better measure of some of the cathode parameters. One can measure eU directly and $\emptyset_{1}$ we have deduced to be $1.5 \pm 0.1$ e.v. for $\mathrm{Al} / \mathrm{Al}_{2} \mathrm{O}_{3}$ by carefully fitting $\mathrm{I}-\mathrm{V}$

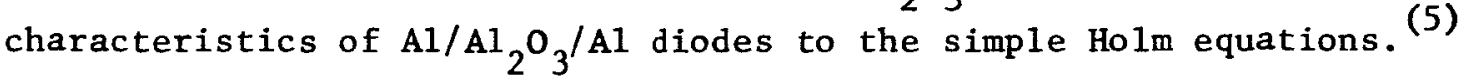
With $\emptyset_{1}$ known, $\emptyset_{2}$ can be determined from the difference in insulator breakdown voltages for opposite polarities, ${ }^{(6)} \mathrm{S}$ can be approximately determined by combining the results of capacitance measurements, interferometry and ellipsometry while $t-s$ can be estimated by careful quartz crystal mass loading techniques. $\emptyset_{3}$ can be measured by the retarding potential method which leaves $L$ and $\ell$ to be determined from the slopes of $\ln \alpha$ vs $s$ and $\ln \alpha$ vs t-S plots. Collins and Davies ${ }^{(7)}$ have deduced values of $\mathrm{L} \approx 5 \AA$ and $l \approx 50 \AA$ from similar measurements.

The computation can be extended without too much trouble to include non-delta function injection, fancier scattering mechanisms and the like but such refinements seem unwarranted in view of the drastic approximations referred to in the first paragraph above. 


\section{SUMMARY}

The most promising cold cathode configuration found to date is an $\mathrm{Al} / \mathrm{Al}_{2} \mathrm{O}_{3} / \mathrm{Pt}$ structure with palladium bars to help distribute current to the thin platinum film. Smoothness of the substrate is very important and the plasma-grown oxide is built up as gently as possible. Baking the device at various stages during and after its fabrication not only improves the characteristics of unused cathodes but partially restores the emission capability of worn out ones. These cathodes do not seem to deteriorate on exposure to room air and in fact will emit electrons into the air. Deterioration comes only through actual use.

So far continuous operation has not been possible because of rapid deterioration but the lifetime under pulse conditions has been improved from a few minutes to several hours. These cathodes already have an emission capability which is useful for certain applications but the deterioration mechanism must be identified and counteracted before they can be used in a vacuum gage. We believe that the deterioration process can be identified by such techniques as direct electronmicroscopic examination of cathode structures before, after and possibly during operation.

A meaningful prognosis for the future of these cathodes can only be made when the reason for the deterioration has been found. 


\section{REFERENCES}

(1) D. O'Boyle. Observations on Electromigration and the Soret Effect in Tungsten, J. App1. Phys. 36, 2849-53 (1965).

(2) F. W. Sears. Thermodynamics, Addison-Wesley (1955) p. 335.

(3) D. Bohn. Quantum Theory, Prentice=Ha11 (1951) p. 278.

(4) J. J. Quinn. Range of Excited Electrons in Metals, Phys. Rev. $126,1453-7(1962)$.

(5) R. Holm. The Electric Tunnel Effect Across Thin Insulator Films in Contact, J. App1. Phys. 22, 569-74 (1951).

(6) J. G. Simmons, Intrinsic Fields in Thin Insulating Films Between Dissimilar Electrodes, Phys. Rev. Lett. 10, 10-12 (1963).

(7) R. E. Collins and L. W. Davies. The Transport of Hot Electrons in $\mathrm{Al}-\mathrm{Al}_{2} \mathrm{O}_{3}-\mathrm{Al}$ Tunnel Cathodes, Solid State Electronics, $\underline{7}$, 445-53 (1964). 


\section{APPENDIX}

The plasma oxidation technique consists of immersing the material to be oxidized in the luminous positive column of a glow discharge which is maintained in about 50 microns pressure of dry oxygen between the base plate and an aluminum ring cathode at the top of the bell jar. The discharge voltage and current will vary with vacuum system geometry but in our system is typically 800 - 1,000 volts, 50-100 milliamps. Since the glow discharge desorbs water from the walls of the system, we maintain a continuous flow of oxygen through the system and also keep a cold finger in the bell jar filled with liquid nitrogen during the oxidation period.

Two methods are used to control the oxide thickness. If a thickness of less than about $40 \AA$ is desired, the sample is merely immersed in the plasma with no electrical connection. It assumes a floating potential of several volts negative with respect to the discharge anode and will grow a high quality amorphous oxide whose thickness is determined by the oxidation time. The range of useful times is about two to thirty minutes.

Thicker oxides are obtained by making electrical contact to the sample and applying a positive bias with respect to the discharge anode. The oxide growth is about $15-20 \AA$ per applied volt.

Certain precautions must be observed in order to obtain the highest quality of oxide. A substrate temperature rise of $20-30^{\circ} \mathrm{C}$ appears to be sufficient to degrade the oxide quality considerably, especially for the thicker oxides, so we usually cool the substrate slightly by placing a brass plate at liquid nitrogen temperature near the back of the substrate. When growing thicker oxides, it appears advantageous to first 
oxidize for a few minutes without making connection to the sample and then to either apply the desired potential gradually or through a high impedance supply so as to limit the anodization rate. If the wire or probe used to make contact with the sample is carefully insulated so that it does not draw any current from the discharge, the current drawn by the sample when it is biased can be observed to decay as the anodization proceeds and indicates when the process has gone to completion. 